\title{
An aphid host-responsive RNA transcript that migrates systemically in plants promotes aphid reproduction
}

\author{
Yazhou Chen ${ }^{1 \$}$, Archana Singh ${ }^{2 \$}$, Gemy G. Kaithakottill ${ }^{2}$, Thomas C. Mathers ${ }^{1}$, \\ Matteo Gravino ${ }^{1}$, Sam T. Mugford ${ }^{1}$, Cock van Oosterhout ${ }^{3}$, David Swarbreck ${ }^{2}$ and \\ Saskia A. Hogenhout ${ }^{{ }^{*}}$
}

${ }^{1}$ Crop Genetics, John Innes Centre, Norwich Research Park, Norwich, NR4 7UH, UK; ${ }^{2}$ Earlham Institute, and ${ }^{3}$ School of Environmental Sciences, University of East Anglia, Norwich Research Park, Norwich, NR4 7UH, UK.

\$Y.C and A.S. contributed equally to the work.

*To whom correspondence may be addressed:

Saskia A. Hogenhout, Department of Crop Genetics, John Innes Centre, Norwich Research Park, Norwich NR4 7UH, United Kingdom

Email: saskia.hogenhout@jic.ac.uk

ORCID: 0000-0003-1371-5606

Author contributions: S.H., C.v.O. and D.S. acquired funding; Y.C., A.S., C.v.O., D.S. and S.H. designed research; Y.C., A.S., G.K., T.M., M.G. and S.M. performed research; Y.C., A.S., G.K. and T.M. analysed data; Y.C., A.S., M.G. and S.H. wrote the original draft of the paper; and Y.C., A.S., T.M., M.G., S.M., C.v.O., D.S. and S.H. reviewed and edited the paper.

The authors declare no conflict of interest.

Data deposition: RNAseq data of $M$. persicae on nine plant species and feeding sites and the genome-guided transcriptome assembly and gene annotations reported in this paper were deposited in the Gene Expression Omnibus (GEO) database, www.ncbi.nlm.nih.gov/geo (SuperSeries accession no. GSE129669, SubSeries accession no. GSE129667, GSE129668). 


\section{ABSTRACT:}

Aphids are sap-feeding insects that colonize a broad range of plant species and often cause feeding damage and transmit plant pathogens, including bacteria, viruses and viroids. These insects feed from the plant vascular tissue, predominantly the phloem. However, it remains largely unknown how aphids, and other sap-feeding insects, establish intimate long-term interactions with plants. To identify aphid virulence factors, we took advantage of the ability of the green peach aphid Myzus persicae to colonize divergent plant species. We found that a $M$. persicae clone of near-identical females establishes stable colonies on nine plant species of five representative plant eudicot and monocot families that span the angiosperm phylogeny. Members of the novel aphid Ya family are differentially expressed in aphids on the nine plant species, are co-regulated and organized as tandem repeats in aphid genomes. Interestingly, aphids translocate $Y a$ transcripts into plants and some transcripts migrate systemically within several plant species. RNAi-mediated knock down of Ya genes reduces $M$. persicae fecundity and $M$. persicae produces more progeny on transgenic plants that heterologously produce one of the systemically migrating Ya transcripts as a long non-coding (Inc)RNA. Taken together, our work led to the discovery of a new host-responsive aphid gene family that operate as virulence factors. Transcripts of this family translocate into plants, including a IncRNA that migrates systemically and promotes aphid reproduction.

Keywords: Plant-insect interactions, aphids, insect vector, effector, virulence, IncRNA, transkingdom RNA trafficking, systemic transport, phloem, polyphagy, generalist.

\section{Significance Statement}

The green peach aphid Myzus persicae causes yield losses of a diverse range of economically important crops primarily as a vector of more than 100 different plant pathogens. We found that a single genotype of $M$. persicae is able to colonize nine plant species, including diverse dicots and maize, indicating that this aphid is truly polyphagous. Members of a new aphid Ya family undergoes coordinated expression changes in $M$. persicae depending on the plant species. The aphids translocate $\mathrm{Ya}$ transcripts into plants during feeding and these RNAs migrate to systemic leaves. 
Moreover, heterologous in planta expression of M. persicae Ya1 as a long non-coding RNA promotes aphid reproduction. Our findings indicate that cross kingdom deployment of RNA is more common than thought.

\section{Introduction}

Sap-feeding insects of the order Hemiptera include aphids, whiteflies, leafhoppers and planthoppers that have piercing-sucking mouthparts, named stylets, for feeding. Many species cause direct feeding damage, known for example as hopper burn $(1,2)$, though global economic yield losses caused by these insects are most often due to their abilities to transmit a diverse range of plant pathogens that include viruses, bacteria and plasmodium-like organisms, and also naked RNA molecules known as viroids $(3,4,5-7,8)$. The majority of insect herbivores are specialized to feed on one or a few closely related plant species (9). Nonetheless, some hemipteran insects are polyphagous. This includes the green peach aphid Myzus persicae, which is known to reproduce on over 400 plant species, and is also able to transmit divergent plant pathogens, including $>100$ plant viruses (10) and the potato spindle viroid (11). The factors involved in the ability of sap-feeding insects to establish intimate interactions with their plant hosts remain still largely unknown.

Sap-feeding insects of the order Hemiptera have stylets as mouthparts that are specialized to feed on plant sap, often from the phloem or xylem of the plant vascular tissue. The way by which sap-feeding insects move their stylets within plant tissues to reach the vascular tissues is arguably best investigated for aphids. These insects establish a long-term feeding site in the phloem sieve cells. However, before reaching the phloem, the stylets probe epidermis, mesophyll and other cells with each probe consisting of a short period of cell content ingestion, often referred to as 'tasting', followed by a short period of salivation (12). As soon as the stylets reach the phloem, aphids deposit saliva into the sieve cells followed by long periods of ingestion of phloem sap (13). The saliva introduced into cells is known as 'watery' saliva and is rich in proteins (14), some of which were shown to be effectors that modulate plant processes (15 - 21). The cycles of 'tasting' and salivation during the stylets path to the phloem are likely to help aphids perceive and adjust to their hosts.

We previously found that a parthenogenetically-reproducing $M$. persicae colony consisting of largely genetically identical females can adjust to the divergent plant species Brassica rapa, Arabidopsis thaliana and Nicotiana benthamiana via 
differential co-regulation of tandemly repeated gene families, including that of Cathepsin $B$ (CathB), which are virulence factors that optimize the ability of $M$. persicae to colonize specific plant species (22). Interestingly, CathB genes are also differentially regulated in aphids on healthy versus those on virus-infected plants (23) and viruses are known to modulate plant defense responses $(24,25)$. These studies provide evidence that $M$. persicae has the ability to perceive the host plant status and adjust its gene expression accordingly.

Here, we build on our previous data, taking advantage of the ability of $M$. persicae to colonize divergent plant species to better understand how aphids colonize plants. We show that a single asexually-reproducing $M$. persicae clone forms stable colonies on nine plant species from five plant families. Via weighted gene co-expression network analysis (WGNCA) (26), we identified a novel gene family, named the Ya family, of which members adjust gene expression in a coordinated manner in response to the different plant species and that are organized as tandem repeats in the $M$. persicae genome. Ya transcripts translocate into plants during aphid feeding and migrate systemically, and the $M$. persicae Ya1 transcript promotes aphid fecundity as a long non-coding (Inc) RNA when produced in plants. Our work shows evidence that the establishment of parasitic interactions between divergent organisms involves the translocation of a IncRNA virulence factor.

\section{Results}

M. persicae clone $\mathrm{O}$ establishes stable colonies on nine plant species that span the Angiosperm phylogeny

To establish $M$. persicae colonies on divergent plant hosts, we selected plant species across representative plant families across the Angiosperm phylogeny (27), including $B$. rapa $(\mathrm{Br})$ and $A$. thaliana $(\mathrm{At})$ of the Brassicaceae, N. benthamiana $(\mathrm{Nb})$ and Solanum tuberosum (St) of Solanaceae, Pisum sativum (Ps) and Phaseolus vulgaris (Pv) of Fabaceae, Helianthus annuus ( $\mathrm{Ha}$ ) and Chrysanthemum indicum (Ci) of Asteraceae, and the monocot plant species, Zea mays $(\mathrm{Zm})$ of the Poaceae (Fig. 1A). Individuals of asexually reproducing females of $M$. persicae clone $O$ that were maintained on $\mathrm{Br}$ for at least three years were transferred to $\mathrm{Br}$ (control) and $\mathrm{At}, \mathrm{Nb}$, St, Ps, Pv, Ha, Ci, and Zm (Fig. 1A). M. persicae clone O achieved 100\% survival rates and established stable colonies on these plant species (Fig. 1B; Suppl. Fig. S1A-D). Aphids survived equally well on $\mathrm{Br}$ and At from the start, and took about four 
weeks to achieve $100 \%$ survival rates on $\mathrm{Nb}$, St, Ps, Ci and $\mathrm{Zm}$, and 10 weeks on Pv and $\mathrm{Ha}$.

\section{Genes differentially expressed in $M$. persicae on nine plant species are enriched for salivary gland and candidate long non-coding RNAs}

Next, we generated transcriptomes of the stable $M$. persicae colonies on the nine plant species. RNA-seq data of five independent biological replicates per plant species were combined with previous RNA-seq data (LIB1777, 22) to give a total of 1.38 billion RNA-seq reads that were assembled into transcripts using a genome-guided approach (Suppl. Table S1; GSE129667). We identified in total 45972 transcripts (corresponding to 19556 genes) with 30127 (65.5\%; 18529 genes) annotated previously (22) and 15845 (34.5\%) novel transcripts (Suppl. Fig. S2A). Of the 45972 transcripts, 6581 transcripts (3 025 genes) had low protein-coding potentials and were assigned candidate IncRNAs (Suppl. Fig. S2B).

Relatively to the M. persicae colonies on $\mathrm{Br}$ (original host), 2490 (1 984 genes) of the 45972 transcripts (5.41\%) were significantly differentially expressed (DE) in the aphids on at least one or more of the other 8 host plant species (fold change $\geqslant 2, p$ value $\leqslant 0.05$, FDR $\leqslant 5 \%$ ) (Suppl. Table S2) and the majority of these show hostspecific expression patterns (Fig. 1C). The DE transcripts were enriched in functions of oxidation-reduction processes, proteolysis (including CathB), and sensory perception of taste (Suppl. Fig. S3). Based on a mean gene expression value of TPM $>5$ across five biological replicates per hosts, 11824 genes (out of 19556 total) were selected for WGCNA, which identified 77 co-expression modules comprising 7864 genes (Suppl. Fig. S4; Suppl. Table S3). Of the 1984 DE genes, 1364 (68\%) were included among the genes of the 77 co-expression modules, and 13 modules were enriched for DE genes (313 DE genes of the total DE genes (16\%); Fisher exact test, $p$ value < 0.05) (Fig. 1D; Suppl. Table S3). Heatmaps based on normalized TPM values of these 13 modules showed different expression patterns in aphids depending on the plant host species (Fig. 1D; Suppl. Fig. S5), suggesting that M. persicae coordinates the expression of groups of genes in response to the plant species.

Remarkably, the darkslateblue, darkorange2, lightcoral, and thistle2 modules among the 13 enriched for DE genes were also enriched for candidate IncRNAs (Fig. 1E; Suppl. Table S3). As well, 8 out of 13 modules, including the darkslateblue, were 
enriched for M. persicae genes expressed in the salivary glands (28) (Fig. 1E; Suppl. Table S3) and 3 out of 13 modules were enriched for gut-expressed genes (Suppl. Fig. S6; Suppl. Table S3). We also noticed that 12 out of 13 modules included genes that lie adjacently to each other as tandem repeats within the aphid genome (Suppl. Table S3). The number of gene repeats varied from 2 to 8 , with the latter being in the darkslateblue module. Hence, different attributes were enriched in the darkslateblue module, including expression in the salivary glands, candidate IncRNAs and tandemly repeated genes. The darkslateblue module is the only one among the 77 modules that contained two groups of genes with exact opposite host-responsive expression patterns (Fig. 1F).

\section{M. persicae preferentially translocates RNA transcripts of DE and candidate IncRNA genes into plants}

Given the enrichment of differentially expressed salivary gland genes that encode candidate IncRNAs, we investigated if these transcripts translocate into plants when aphids feed. Leaves of 4-week-old $A$. thaliana plants were caged with 20 adult aphids (feeding site) or no aphids (control) for 24 hours (Fig. 2A; Suppl. Table S4). Reads that uniquely aligned to the $M$. persicae genome were identified, and at TPM $\geq$ 50, these corresponded to between 1837 to 3154 aphid transcripts, depending on the biological replicate, in leaf sections exposed to aphids only (Suppl. Table S5). Based on the presence in at least three biological replicates, $3186 \mathrm{M}$. persicae transcripts of which 201 are candidate IncRNAs were found present in feeding sites (Fig. 2A). The candidate IncRNAs were enriched among the transcripts found in the feeding site (Fisher's Exact Test, $p=1.02 E-48$ ) (Fig. 2A; Suppl. Table S4). The candidate IncRNAs in the feeding site belonged to ten co-expression modules that were enriched for DE genes, including the darkslateblue module (Fig. 2B). Moreover, the $M$. persicae transcripts in the feeding site were enriched for DE genes and salivary gland transcripts (Fisher's Exact Test, $p=3.7 \mathrm{E}-13$ and $p=0.03$, respectively) and in both categories the transcripts were also enriched for candidate IncRNAs (Fisher's Exact Test, $p=0.002$ and $p=3.8 \mathrm{E}-35$, respectively) (Fig. 2C). Therefore, $M$. persicae may preferentially translocate candidate IncRNAs of salivary gland expressed genes that are DE in aphids on divergent hosts into feeding sites. 
The co-regulated $M$. persicae genes of the darkslateblue module includes all 30 members of the Ya family tandem repeat

Upon further analyses of genes in the darkslateblue module, 37 out of total of 56 genes were found to encode candidate IncRNAs. Of these 37, 23 were identified to have high sequence similarities $( \pm 80 \%)$ and located in close proximity to one another on six scaffolds. These genes were organized in series of tandem repeats with less than 1-kb distance between the gene copies. Given these characteristics, the genes appear to belong to a gene family.

Tandemly duplicated genes with highly similar sequences are often misannotated due to incorrect alignment of RNA-seq reads. Therefore, we manually this gene family by searching via BLAST the entire $M$. persicae genome for regions that align to a 148-bp nucleotide sequence that was found to be conserved among the 23 candidate IncRNA genes (Suppl. Fig. S7). This resulted in the identification of 7 additional genes to make a 30-member gene family (Suppl. Table S6). We updated the transcriptome assembly for this gene locus with the manually annotation (GSE129667). We named this gene family the Ya family (Yá means aphid in Chinese).

The 3' ends of the 30 Ya transcripts were manually corrected based on the presence of a poly(A) signal and 5' ends via identification of conserved sequences among the Ya transcripts. All Ya genes have a three-exon structure, and they show a modest to high sequence conservation (ranging between 84.6 - 99.1\% nucleotide identities compared to Ya1), including a region that corresponds to a small open reading frame (ORF) that may translate into a peptide of 38 amino acids in all $30 \mathrm{Ya}$ transcripts (Fig. 3A; Suppl. Fig. S8).

Phylogenetic analyses based on nucleotide sequence alignment (Suppl. Fig. S8) group the Ya genes into roughly 4 distinct clades (Fig. 3B). As well, we confirmed that the Ya genes form several tandem repeats in the $M$. persicae genome in which the Ya genes were often, but not always, organized in pairs facing outwards on opposite genomic strands (Fig. 3C). The pairs belong to the same or different phylogenetic clusters of the Ya phylogenetic tree. Repeating the M. persicae nine-host swap WGCNA analysis with the new set of manually annotated $30 \mathrm{M}$. persicae Ya genes grouped all Ya members within the same (darkslateblue) co-expression module (Suppl. Fig. S9), as before, suggesting that the 30 Ya genes are co-regulated.

Ya genes were also found in five other aphid species for which whole-genome sequences are available. To further compare the number of Ya genes among aphid 
species, the 148-bp Ya1 exon 2 sequence was BLAST searched against genome assemblies of Aphis glycines, Acyrthosiphon pisum, Diuraphis noxia, M. cerasi and Rhopalosiphum padi and subsequently against public transcriptome datasets for these species. Transcripts that matched the genomic sequences were assigned as $\mathrm{Ya}$ genes, resulting in the identification of 13 candidate Ya genes in Aphis glycines, 17 in Diuraphis noxia, 29 in M. cerasi and 33 in Rhopalosiphum padi. In all five aphid species, Ya genes are tandem repeats in genomes. We did not find Ya genes in genomes of hemipteran insect species beyond aphids.

Therefore, Ya genes are unique to aphids and are part of larger families that form tandem repeats in aphid genomes and are differentially expressed and co-regulated in $M$. persicae on nine divergent host plant species.

\section{Translocated $M$. persicae Ya transcripts migrate systemically in plants}

To asses if aphid Ya transcripts migrate systemically within plants, we caged twenty adult aphids on leaves of 4 -week-old $A$. thaliana plants for $24 \mathrm{hrs}$, and then examined the presence of aphid transcripts in the caged area (feeding site), the area from the petiole to the aphid cage on the leaf (near feeding site) and a systemic leaf (Fig. 4A). We designed specific primers for the nine Ya transcripts found in the feeding site (Suppl. Fig. S10, Suppl. Table S7). For the six Ya transcripts Ya1, Ya2, Ya3, Ya6, Ya11, Ya17 the correct sizes of amplification products were obtained and the sequences of these amplification products matched those of the Ya transcripts, whereas the presence of Ya6, Ya21 and Y23 transcripts was not confirmed (Suppl. Fig. S10). Ya1, Ya2, and Ya17 transcripts were detected in feeding and systemic sites indicating that these transcripts migrated away from the feeding site into systemic tissues (Suppl. Fig. S10). We focused further analyses on the Ya1 transcript, because the heatmap of the darkslateblue module suggested that Ya1 is upregulated in aphids on $\mathrm{Br}$ and At (Suppl. Fig. S9). The predicted size of the Ya1 transcript is $382 \mathrm{nt}$ (Fig. 4B, Suppl. Fig. S11A). The presence of Ya transcripts in aphids and plants were analysed by reverse transcriptase PCRs with a series of specific primers and by northern blot hybridizations with a Ya1 probe. PCRs showed that a 357-nt Ya transcript that starts at nucleotide 25 of exon 1 was detected in the aphid, and not in plants, whereas a shorter Ya1 transcript of 273 nt that starts at nucleotide 110 near the start of the sequence corresponding to exon 2 was detected in the plant and migrated systemically (Fig. 4B, Suppl. Fig. S11A and B). 3' RACE experiments showed that 
the Ya1 transcript has a poly(A) tail (Suppl. Fig. S11C). Northern blots confirmed the sizes of the Ya transcripts in aphids and plants; the fragments that hybridized to the Ya1-SP6 in aphids were larger than the $291 \mathrm{nt}$ Ya1-SP6 transcript, whereas the Ya1 fragments in plants were shorter than this transcript (Fig. 4B). These data indicate that the first \pm 100 nt at the 5 ' end of the 357-nt aphid Ya1 transcript is processed to produce a 273-nt Ya1 transcript upon translocation into plants.

Northern blots and qRT-PCRs showed that the Ya1 transcript gradually decreases in concentration from feeding sites to near feeding sites and systemic leaves of $A$. thaliana (Fig. 4C, 4D, Suppl. Fig. S13). Ya1 also migrated systemically in B. rapa, $P$. sativum and Z. mays exposed to M. persicae (Fig. 4E, Suppl. Fig. S12). Sequencing of the PCR products derived from of the feeding sites, near feeding sites and systemic leaves of these three hosts revealed identical sequences to Ya1, which is different from the other Ya family members (Suppl. Fig. S14). Therefore, M. persicae deposits Ya1 transcripts into four divergent host plant species during feeding and these transcripts migrate systemically within these plants away from aphid feeding sites.

\section{M. persicae Ya1 is a virulence factor}

Differential expression of Ya1 in M. persicae among hosts and migration of Ya1 in various of hosts suggest Ya1 may play roles in aphid-host interactions. To investigate this, we generated transgenic lines expressing dsRNA corresponding to the Ya1 sequence for plant-mediated RNAi of Ya1 in M. persicae. Two independent A. thaliana lines 1-5 and 2-8 successfully knocked down Ya1 expression compared to WT Col-0 and lines producing dsRNA corresponding to GFP (dsGFP) in M. persicae (Fig. 5A). M. persicae on $A$. thaliana lines 1-5 and 2-8 had reduced fecundity compared to WT Col-0 and dsGFP lines (Fig. 5B). Therefore, knock down of the expression of Ya1 reduces $M$. persicae reproduction on $A$. thaliana.

Given that aphids deposit Ya1 into plants and that this RNA migrates systemically, we investigated if stable expression of Ya1 in planta affects $M$. persicae performance. We generated stable transgenic plants that produced the 273-nt (exons 2 + 3) Ya1 transcript (35S::Ya1 (Col-0) lines 8-8 and 9-9) and 273-nt Ya1 mutants in which three ATG start sites within the 38-aa open reading frame were mutated to stop codons (35S::Ya1_mut (Col-0) lines 1-1, 3-3 and 4-4) (Fig. 5C and D, Suppl. Fig. S15). M. persicae produced more progeny on both 35S::Ya1 and 35S::Ya1_mut 
compared to 35S:GFP and Col-0 plants (Fig. 5E, Suppl. Fig. S16), indicating that the Ya1 RNA transcript modulates plant processes that leads to an increase of $M$. persicae fecundity. Taken together, our data show that M. persicae translocate the Ya transcript into plants during feeding and this transcript migrates systemically within plants and promotes fecundity of this aphid. Therefore, Ya1 is an aphid IncRNA virulence factor.

\section{Discussion}

We found that progeny derived from a single asexually-reproducing $M$. persicae clone $\mathrm{O}$ female stably colonizes nine divergent plant species of five families that span the Angiosperm phylogeny (27), demonstrating that $M$. persicae is truly polyphagous. We identified $M$. persicae genes that show coordinated up- and down-regulation depending on the plant species the aphid is colonizing and that are organized as repeats in the aphid genome. The genes are organized in co-expression modules of which 13 are enriched for DE genes, genes expressed in the salivary glands and candidate IncRNAs. One of these modules includes all members of the Ya family with 30 genes that are tandemly repeated in the M. persicae genome. Moreover, transcripts from six Ya genes translocate into plants during aphid feeding and three migrate systemically away from the aphid feeding site to distant leaves. Via RNAi-mediated knock down of Ya expression we show evidence that Ya genes contribute to aphid performance on $A$. thaliana and are therefore virulence factors. Heterologous expression of the Ya1 transcript without the small putative ORF in planta promotes aphid performance indicating that the Ya1 RNA transcript acts as a IncRNA virulence factor.

Because genome annotation pipelines are generally focused on protein-coding genes, the majority Ya family members were not annotated in the first version of the M. persicae genome annotation (22). Moreover, due to the Ya family being organised as tandem repeats of highly conserved sequences, genome-guided transcript assembly generated misassembled transcripts. To overcome this we did thorough manual annotation of the Ya region and were able to identify the 30-member Ya family, characterize the expression patterns of each of the family members in $M$. persicae in response to the nine plant species, and to assess which family members translocate to plants during aphid feeding. We show that $M$. persicae Ya members are characterized by a 3-exon gene model and produce transcripts ranging from 357 403 nucleotides in lengths. When analysing other hemipterans, we identified tandemly 
repeated Ya family members only in the genomes of only other aphid species among hemipterans for which genome sequence data are available, suggesting that these IncRNAs are unique to aphids.

Despite Ya genes being annotated as genes with low-coding potential, Ya transcripts have a conserved small ORF that potentially translates into a 38 amino acid peptide. Mutations that prevent translation of this peptide within the Ya1 transcript in plants does not affect the ability of Ya1 to promote aphid fecundity, indicating that the Ya1 has virulence activity as a IncRNA. ORFs have been detected in number of transcripts known to function as IncRNAs, including RNAs associated with ribosome function $(30 ; 31)$ and $X$-inactive specific transcript (Xist) that regulates the $X$ chromosome inactivation process (32), and it is being debated whether IncRNA ORFs may be translated in some situations (33). Whether the small ORF found to be conserved across Ya genes has a function remains to be determined, but based on our annotations and functional analyses of $Y a 1$, at least one member of the Ya gene family appears to function as a IncRNA.

Several parasites translocate small RNAs into their hosts (34 - 36). The functions of these transkingdom small RNAs are known for only a few parasites. For example, small RNAs of the fungal plant pathogen Botrytis cinerea interact with the AGO1 protein of the $A$. thaliana RNA interference (RNAi) machinery to suppress plant defense genes (37) and microRNAs of the parasitic plant dodder target host messenger RNAs involved in plant pathogenesis (38). In the opposite direction, plants export specific microRNAs to control virulence of a pathogenic fungus (39). In some parasite-host interactions, a large number of long transcripts (>200 nt) were found in hosts, including RNA transcripts of Cryptosporidium parvum in the nuclei of human intestinal epithelial cells (40) and transcripts of dodder that systemically migrate in Solanum lycopersicum and $A$. thaliana (41). However, it is unclear if these larger parasite RNAs modulate parasite-host interactions. Here we show that $M$. persicae RNA transcripts translocate into plants. These include transcripts of the Ya family that migrate systemically. Knock down of Ya gene expression via RNAi reduces aphid fecundity, whereas in planta expression of Ya1 as a IncRNA promotes aphid fecundity. This suggests that the aphid Ya genes are virulence factors and that the Ya1 transcript controls aphid performance via the plant.

The M. persicae Ya1 IncRNA may be an effector that modulates specific plant processes. Interestingly, the entire $M$. persicae Ya family of 30 members is part of the 
darkslateblue module, which is the only module among the 77 co-expression modules that consists of two groups of genes with exact opposite expression patterns. The module also includes several protein-coding genes with similarities to haemolymph juvenile hormone binding and WD40 and EGF-like domain-containing proteins that have roles in signal transduction in insects. Given this finding, a non-mutually exclusive possibility is that Ya1 and other Ya IncRNAs may have a sensing role within aphids; for example, IncRNAs not degraded in plants may migrate back into the aphid to regulate aphid gene expression, in agreement with IncRNAs often have functions in gene expression regulation (42 - 44). Whereas the mechanism by which Ya1 controls aphid fecundity will have to be determined, results described herein indicate that members of the Ya gene family are aphid-specific virulence factors.

\section{Material and Methods: See Supplementary File 1.}

\section{Acknowledgments}

We thank Danielle Goff-Legett, Alexandra Kolodyazhnaya and Christian Aarssen for assisting with various experiments in this study, and Andrew David Lyle Nelson and Upendra Kumar Devisetty at The University of Arizona for their assistance with the initial steps of IncRNA identifications. We are grateful to Anna Jordan, Darrell Bean, Susannah Gill, and lan Bedford of the JIC Entomology Facility for rearing and taking care of aphid colonies and Horticultural Services for growing the plants used in this study. The project was funded by the Biotechnology and Biological Sciences Research Council (BBSRC) Industrial Partnership Award with Syngenta Ltd grant BB/R009481/1 and the BBSRC Institute Strategic Program Grant (ISPG) Plant Health BB/P012574/1.

\section{References}

1. V. G. S. Kaplin, Yu. A. Sharapova, Influence of the Russian wheat aphid Diuraphis noxia (Kurdjumov) (Homoptera, Aphididae) on productive qualities of spring bread wheat and barley grown from the seeds from aphid-infested spikes. Entomol. Rev. 97, 415-424 (2017) . 
2. E. A. Backus, M. S. Serrano, C. M. Ranger, Mechanisms of hopperburn: an overview of insect taxonomy, behavior, and physiology. Annu Rev Entomol 50, 125-151 (2005).

3. M. Heck, Insect transmission of plant pathogens: a systems biology perspective. mSystems. 3, pii: e00168-17 (2018).

4. N. van Bogaert, K. de Jonghe, E.J. van Damme, M. Maes, G. Smagghe, Quantitation and localization of pospiviroids in aphids. J. Virol. Methods. 211, 5154 (2015).

5. E. Jaskowska, C. Butler, G. Preston, S. Kelly, Phytomonas: trypanosomatids adapted to plant environments. PLoS Pathog. 11, e1004484 (2015).

6. Y. Walia, S. Dhir, A. A. Zaidi, V. Hallan, Apple scar skin viroid naked RNA is actively transmitted by the whitefly Trialeurodes vaporariorum. RNA Biol. 12,11311138 (2015).

7. S.A. Hogenhout, el-D. Ammar, A. E. Whitfield, M. G. Redinbaugh, Insect vector interactions with persistently transmitted viruses. Annu. Rev. Phytopathol. 46,327359 (2008).

8. J. C. Ng, B. W. Falk, Virus-vector interactions mediating nonpersistent and semipersistent transmission of plant viruses. Annu. Rev. Phytopathol. 44,183-212 (2006).

9. L. M. Schoonhoven, J. J. A. van Loon, M. Dicke Insect-Plant Biology (OUP Oxford; 2 edition) (2005).

10. CABI, Myzus persicae (green peach aphid). Invasive Species Compendium (2015).

11. J. M. Syller, W. Marczewski, J. Pawłowicz, Transmission by aphids of potato spindle tuber viroid encapsidated by potato leafroll luteovirus particles. Eur. J. Plant Pathol. 103, 285-289 (1997).

12. W.F. Tjallingii, Salivary secretions by aphids interacting with proteins of phloem wound responses. J. Exp. Bot. 57, 739-745 (2006).

13. W. F. Tjallingii, T. H. Esch, Fine-structure of aphid stylet routes in plant-tissues in correlation with EPG signals. Physiol. Entomol. 18, 317-328 (1993).

14. T. Will, A.C. Furch, M. R. Zimmermann, How phloem-feeding insects face the challenge of phloem-located defenses. Front. Plant. Sci. 4, 336 (2013).

15. N.S. Mutti, J. Louis, L. K. Pappan, K. Pappan, K. Begum, M. S. Chen, Y. Park, N. Dittmer, J. Marshall, J. C. Reese, G. R. Reeck, A protein from the salivary glands 
of the pea aphid, Acyrthosiphon pisum, is essential in feeding on a host plant. Proc. Natl. Acad. Sci. U. S. A. 105, 9965-9969 (2008).

16. J. I. Bos, D. Prince, M. Pitino, M. E. Maffei, J. Win, S. A. Hogenhout, A functional genomics approach identifies candidate effectors from the aphid species Myzus persicae (green peach aphid). PLoS Genet. 6, e1001216 (2010).

17. M. Pitino, S. A. Hogenhout, Aphid protein effectors promote aphid colonization in a plant species-specific manner. Mol. Plant Microb Interact. 26,130-139 (2013).

18. G. J. Kettles, I. Kaloshian, The potato aphid salivary effector Me47 is a glutathione-S-transferase involved in modifying plant responses to aphid Infestation. Front. Plant Sci. 7,1142 (2016).

19. S. T. Mugford, E. Barclay, C. Drurey, K. C. Findlay, S. A. Hogenhout, An immunosuppressive aphid saliva protein is delivered into the cytosol of plant mesophyll cells during feeding. Mol. Plant Microbe Interact. 29, 854-861 (2016).

20. P. A. Rodriguez, C. Escudero-Martinez, J. I. Bos, An aphid effector targets trafficking protein VPS52 in a host-specific manner to promote virulence. Plant Physiol. 173,1892-1903 (2017).

21. R. Chaudhary, H. C. Peng, J. He, J. MacWilliams, M. Teixeira, T. Tsuchiya, Q. Chesnais, M. B. Mudgett, I. Kaloshian, Aphid effector Me10 interacts with tomato TFT7, a 14-3-3 isoform involved in aphid resistance. New Phytol. 221,1518-1528 (2019).

22. T. C. Mathers, Y. Chen, G. Kaithakottil, F. Legeai, S. T. Mugford, P. Baa-Puyoulet, A. Bretaudeau, B. Clavijo, S. Colella, O. Collin, T. Dalmay, T. Derrien, H. Feng, T. Gabaldón, A. Jordan, I. Julca, G. J. Kettles, K. Kowitwanich, D. Lavenier, P. Lenzi, S. Lopez-Gomollon, D. Loska, D. Mapleson, F. Maumus, S. Moxon, D. R. Price, A. Sugio, M. van Munster, M. Uzest, D. Waite, G. Jander, D. Tagu, A. C. Wilson, C. van Oosterhout, D. Swarbreck, S. A. Hogenhout, Rapid transcriptional plasticity of duplicated gene clusters enables a clonally reproducing aphid to colonise diverse plant species. Genome Biol. 18, 27 (2017).

23. P. V. Pinheiro, M. Ghanim, M. Alexander, A. R. Rebelo, R. S. Santos, B. C. Orsburn, S. Gray, M. Cilia, Host plants indirectly influence plant virus transmission by altering gut cysteine protease activity of aphid vectors. Mol. Cell Proteomics 16, S230-S243 (2017).

24. K. E. Mauck, Q. Chesnais, L. R. Shapiro, Evolutionary determinants of host and vector manipulation by plant viruses. Adv. Virus Res. 101,189-250 (2018). 
25. J. P. Carr, A. M. Murphy, T. Tungadi, J. Y. Yoon, Plant defense signals: players and pawns in plant-virus-vector interactions. Plant Sci. 279, 87-95 (2019).

26. P. Langfelder, S. Horvath, WGCNA: an R package for weighted correlation network analysis. BMC Bioinformatics 9, 559 (2008).

27. S. D. Gamboa-Tuz, A. Pereira-Santana, T. Zhao, M. E. Schranz, E. Castano, L. C. Rodriguez-Zapata, New insights into the phylogeny of the TMBIM superfamily across the tree of life: Comparative genomics and synteny networks reveal independent evolution of the BI and LFG families in plants. Mol. Phylogenet. Evol. 126, 266-278 (2018).

28. J. S. Ramsey, A. C. Wilson, M. de Vos, Q. Sun, C. Tamborindeguy, A. Winfield, G. Malloch, D. M. Smith, B. Fenton, S. M. Gray, G. Jander, Genomic resources for Myzus persicae: EST sequencing, SNP identification, and microarray design. BMC Genomics 8: 423 (2007).

29. S. A. Mousavi, A. Chauvin, F. Pascaud, S. Kellenberger, E. E. Farmer, GLUTAMATE RECEPTOR-LIKE genes mediate leaf-to-leaf wound signalling. Nature 500, 422-426 (2013).

30. C. Zeng, M. Hamada, Identifying sequence features that drive ribosomal association for IncRNA. BMC Genomics 19, 906 (2018).

31. C. Zeng, T. Fukunaga, M. Hamada, Identification and analysis of ribosomeassociated IncRNAs using ribosome profiling data. BMC Genomics 19, 414 (2018).

32. A. Sahakyan, Y. Yang, K. Plath, The Role of Xist in X-chromosome dosage compensation. Trends Cell Biol. 28, 999-1013 (2018).

33. Z. Ji, R. Song, A. Regev, K. Struhl, Many IncRNAs, 5'UTRs, and pseudogenes are translated and some are likely to express functional proteins. Elife 4, e08890 (2015).

34. J. H. Zhao, H. S. Guo HS, Trans-kingdom RNA interactions drive the evolutionary arms race between hosts and pathogens. Curr Opin Genet Dev. 58-59, 62-69 (2019).

35. P.J. van Kleeff, M. Galland, R.C. Schuurink, P. M. Bleeker, Small RNAs from Bemisia tabaci are transferred to Solanum lycopersicum phloem during feeding. Front Plant Sci. 7,1759 (2016) 2016.

36. T. Meningher, G. Lerman, N. Regev-Rudzki, D. Gold, I. Z. Ben-Dov, Y. Sidi, D. Avni, E. Schwartz, Schistosomal MicroRNAs isolated from extracellular vesicles 
in sera of infected patients: a new tool for diagnosis and follow-up of human Schistosomiasis. J Infect Dis. 215, 378-386 (2017).

37. Weiberg A, M. Wang, F. M. Lin, H. Zhao, Z. Zhang, I. Kaloshian, H. D. Huang, H. Jin, Fungal small RNAs suppress plant immunity by hijacking host RNA interference pathways. Science 342, 118-123 (2013).

38. S. Shahid, G. Kim, N. R. Johnson, E. Wafula, F. Wang, C. Coruh, V. BernalGaleano, T. Phifer, C. W. dePamphilis, J. H. Westwood, M. J.Axtell, MicroRNAs from the parasitic plant Cuscuta campestris target host messenger RNAs. Nature 553, 82-85 (2018).

39. T. Zhang, Y. L. Zhao, J. H. Zhao, S. Wang, Y. Jin, Z. Q. Chen, Y. Y. Fang, C. L. Hua, S. W. Ding, H. S. Guo, Cotton plants export microRNAs to inhibit virulence gene expression in a fungal pathogen. Nat Plants. 2,16153, (2016)

40. Y. Wang, A. Y. Gong, S. Ma, X. Chen, Y. Li, C. J. Su, D. Norall, J. Chen, J. K. Strauss-Soukup, X. M. Chen, Delivery of parasite RNA transcripts into infected epithelial cells during Cryptosporidium infection and its potential impact on host gene transcription. J Infect Dis. 215, 636-643 (2017).

41. G. Kim, M. L. LeBlanc, E. K. Wafula, C. W. dePamphilis, J. H. Westwood, Genomic-scale exchange of mRNA between a parasitic plant and its hosts. Science. 345, 808-11 (2014).

42. A. Di Ruscio, A. K. Ebralidze, T. Benoukraf, G. Amabile, L. A. Goff, J. Terragni, M. E. Figueroa, L. L. De Figueiredo Pontes, M. Alberich-Jorda, P. Zhang, M. Wu, F. D'Alò, A. Melnick, G. Leone, K. K. Ebralidze, S. Pradhan, J. L. Rinn, D. G. Tenen, DNMT1-interacting RNAs block gene-specific DNA methylation. Nature 503, 371-376 (2013).

43. B. K. Sun, A. M. Deaton, J. T. Lee, A transient heterochromatic state in Xist preempts $X$ inactivation choice without RNA stabilization. Mol. Cell. 21, 617-628 (2006).

44. K. M. Schmitz, C. Mayer, A. Postepska, I. Grummt, Interaction of noncoding RNA with the rDNA promoter mediates recruitment of DNMT3b and silencing of rRNA genes. Genes Dev. 24, 2264-2269 (2010). 


\section{Figure legends}

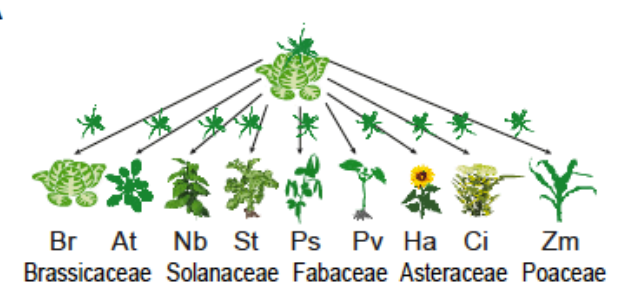

C

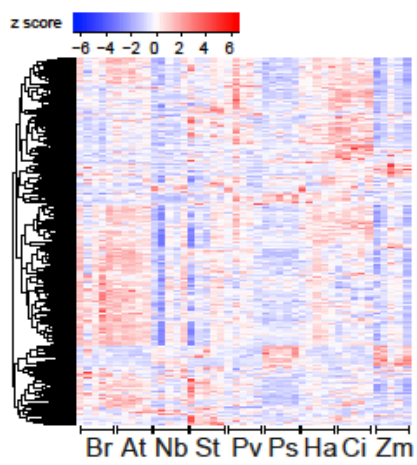

E

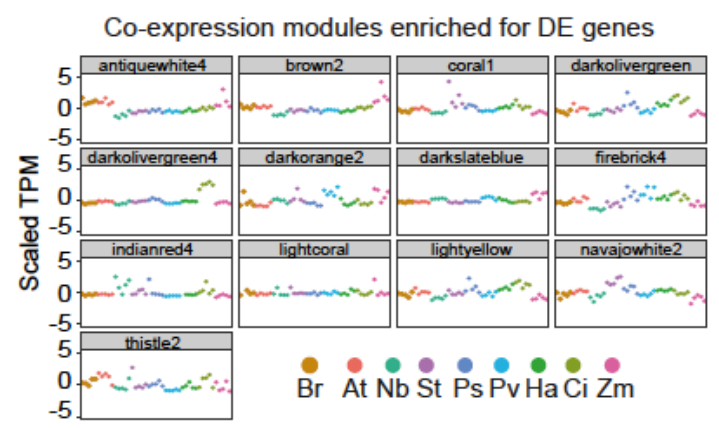

B

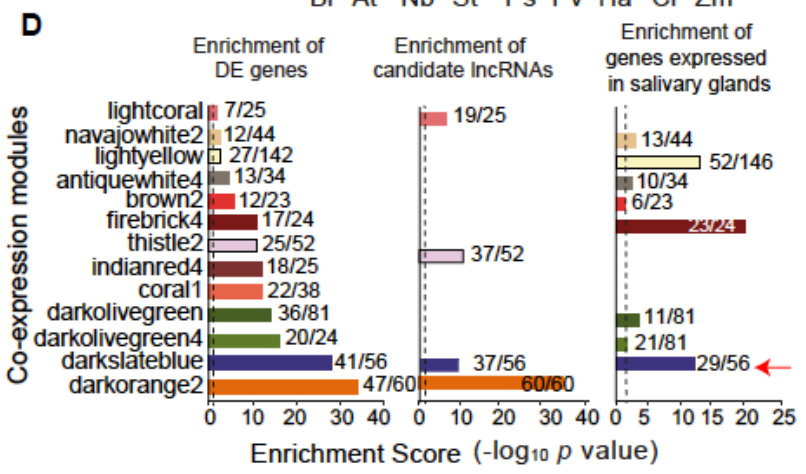

F Expression of genes in darkslateblue

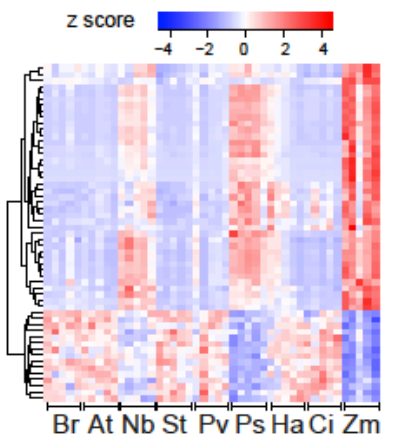

Fig. 1. Colonisation of M. persicae on divergent host plant species involves coexpression of genes enriched in salivary gland expression and that encode IncRNAs. (A) Schematic overview of the experimental setup. B. rapa (Br), A. thaliana (At), N. benthamiana (Nb), S. tuberosum (St), C. indicum (Ci), H. annuus (Ha), P. sativum $(\mathrm{Ps}), P$. vulgaris $(\mathrm{Pv})$ and Zea mays $(\mathrm{Zm})$. (B) Reproduction levels of stable $M$. persicae colonies on each of the nine plant species as shown in A. Box plots show mean numbers of nymphs produced over the lifespan of an adult female on each plant species. (C) Heatmaps of log transformed TPM values of 1984 DE genes of $M$. persicae colonies on $\mathrm{Br}$ versus colonies on one or more of the other 8 plant species as shown in A at 5 biological replicates per host. (D) Enrichment scores of 13 WGNCA modules. The $x / y$ above the bars indicating gene numbers in the enriched category $(x)$ and module $(\mathrm{y})$. Dashed vertical lines, enrichment score $2(p$ value $=0.01)$. Red arrow, darkslateblue module. (E) Scatter plots of 13 WGCNA modules enriched for DE genes. (F) Heatmap of log transformed TPM values of genes in the darkslateblue module. 
A

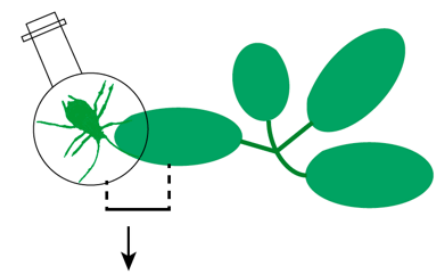

-> Remove aphids and thoroughly wash of leaf -> RNA-seq
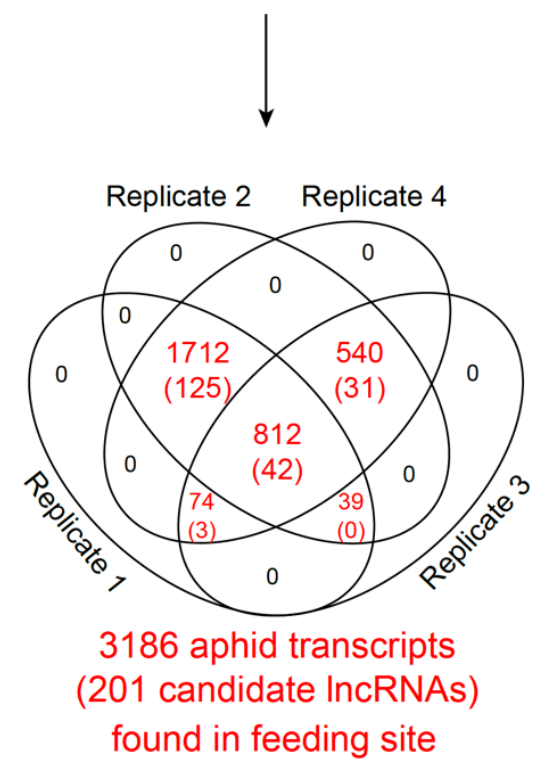

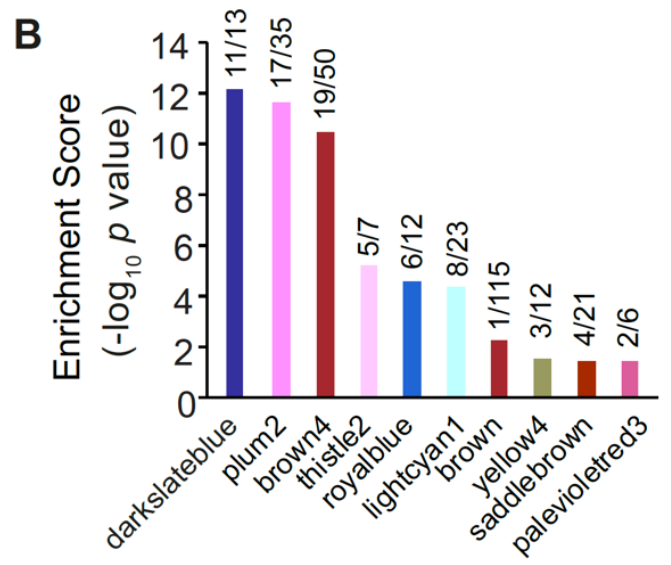

C

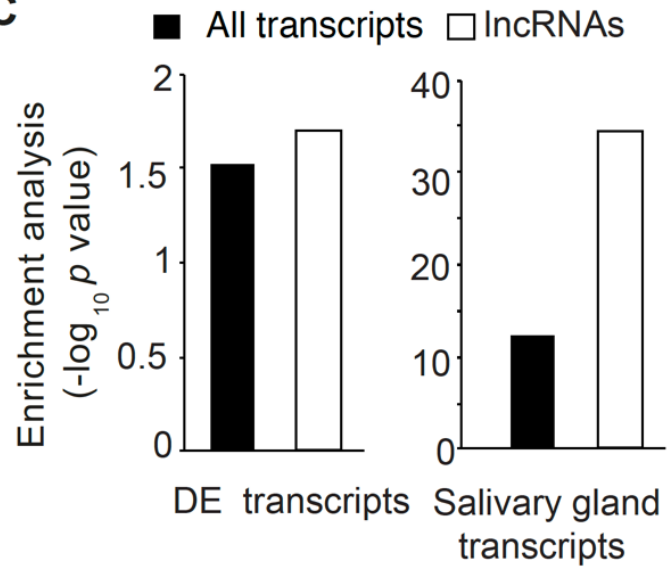

Fig. 2. Aphid transcripts at the aphid feeding site are enriched for differential expression in aphids on nine plant species, candidate IncRNA and expression in aphid salivary glands. (A) Schematic overview of the experimental setup. Venn diagram shows the number of aphid transcripts found in plants at the aphid feeding site at TPM $\geq 50$ in one of the replicates and/or presence of transcripts in at least three biological replicates. (B) Transcripts in the feeding site were enriched for candidate IncRNAs in 10 out of the 13 co-expression modules enriched for DE genes (see Fig. 1D); $x / y$ above the bars indicating IncRNAs $(x)$ and the total number of transcripts that belong to the module found in the feeding site (y). (C) Enrichment of genes that are DE (left graph) and expressed in salivary glands (28) (right graph) of aphid transcripts found in the plant at the aphid feeding. Black bars, all aphid transcripts. White bars, candidate IncRNAs. 
A

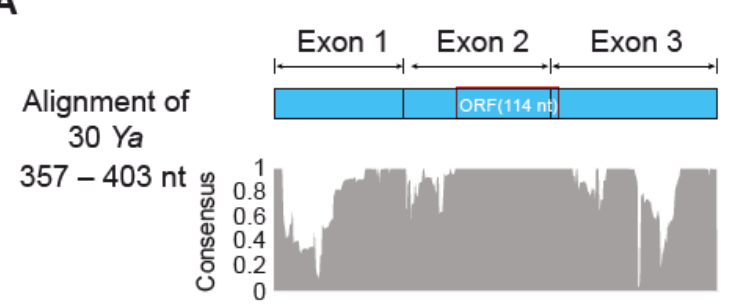

C Scaffold 667 (83971-130747) Genome - 2kb

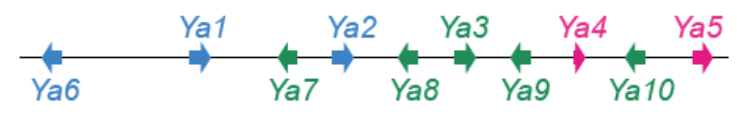

Scaffold 864 (7162-57788)
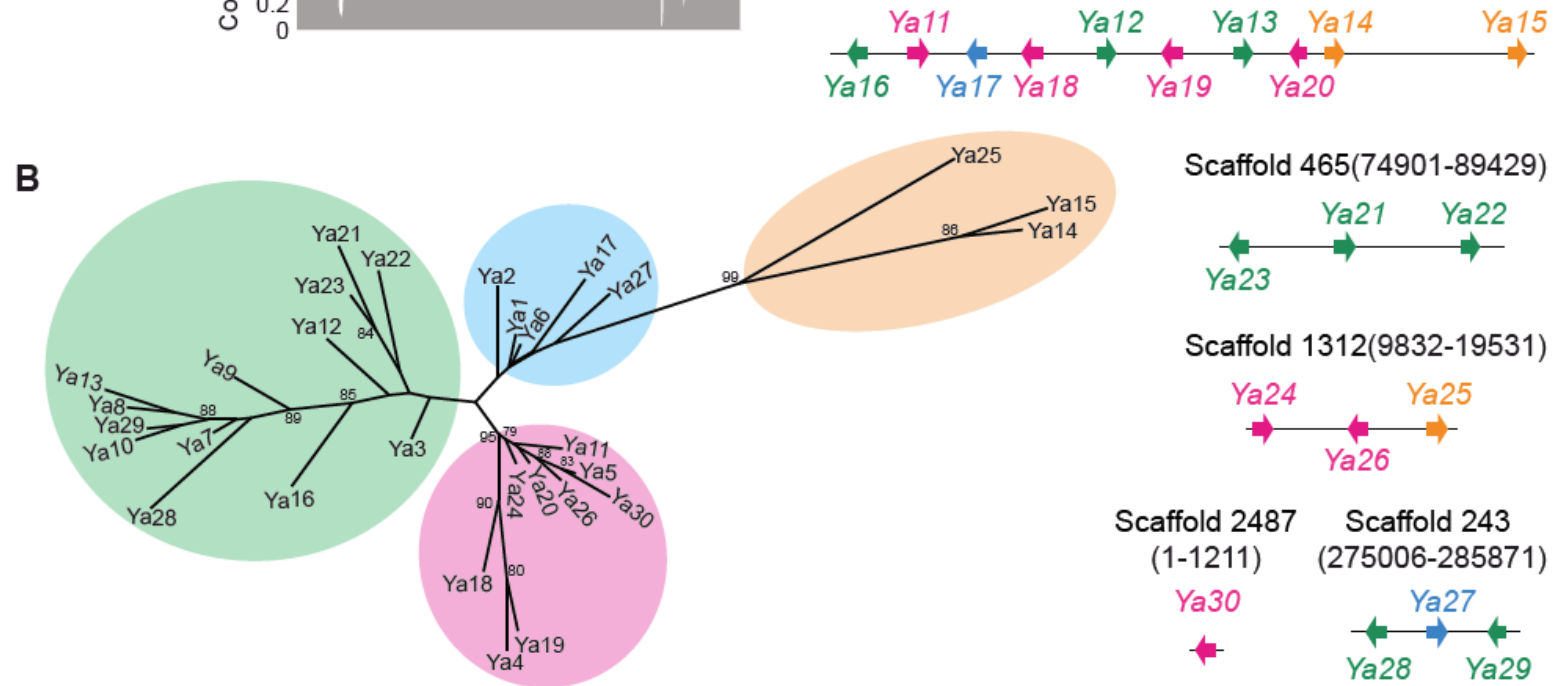

Scaffold 465(74901-89429)

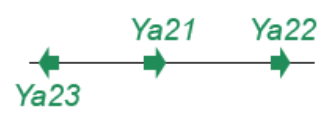

Scaffold 1312(9832-19531)

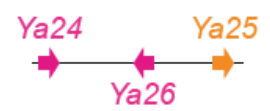

Scaffold 2487 Scaffold 243

$(1-1211) \quad(275006-285871)$

Yазо

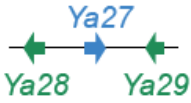

Fig. 3. Annotation of the Ya gene family in the $M$. persicae genome. (A) Level of conservation (consensus) among aligned nucleotide sequences of mature transcripts of 30 Ya genes (See Suppl. Fig. S8 for alignment). The small open reading frame (ORF) may translate into a 38 amino acid (aa) peptide. (B) Phylogeny based on the alignment of 30 Ya transcripts shown in A. (C) Locations of Ya gene repeats on $M$. persicae genomic scaffolds. Ya genes are shown as box arrows of which the colours match the clades of the Ya phylogenetic tree shown in B. 


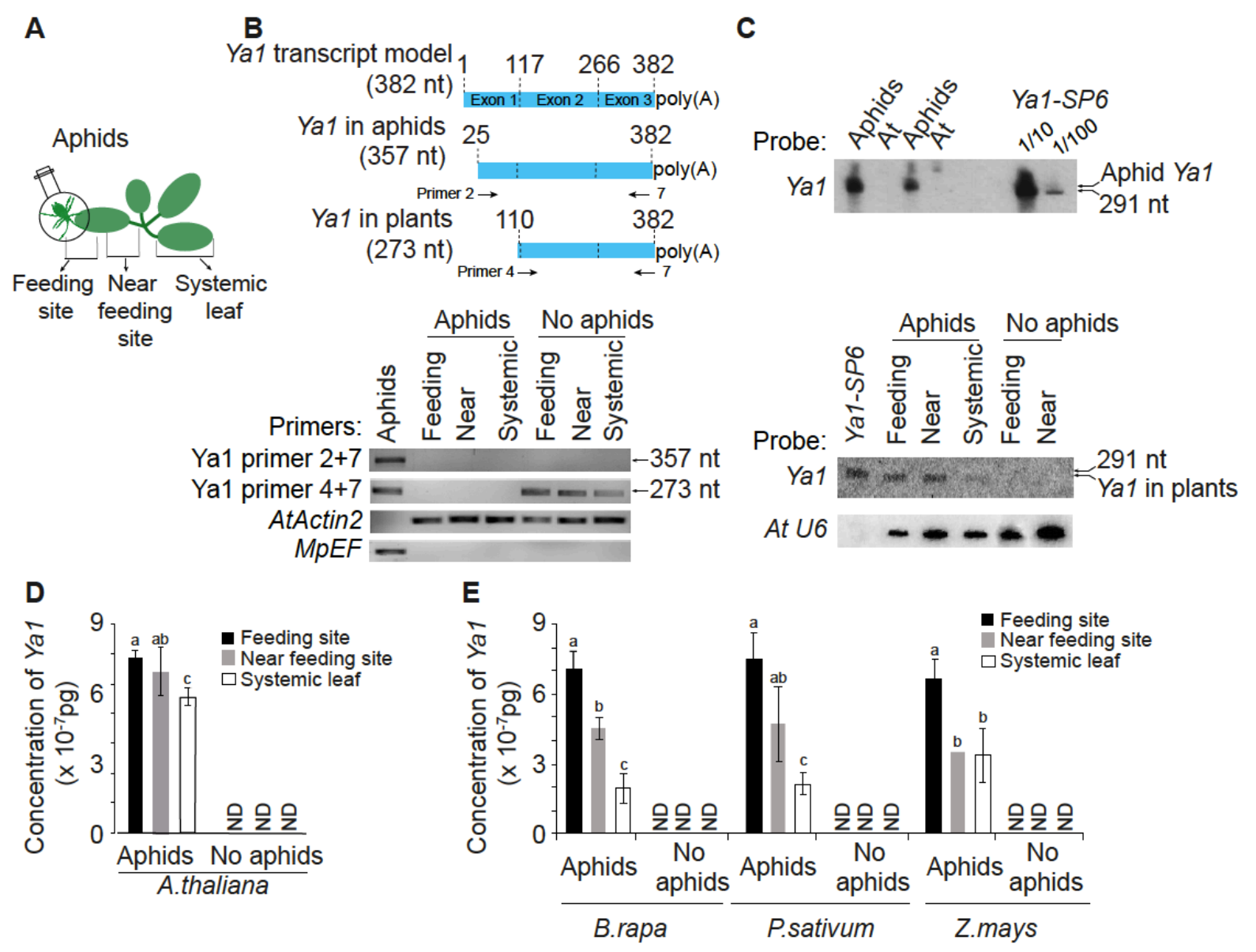

Fig. 4. The M. persicae Ya1 transcript translocates and systemically migrates in plants. (A) Schematic overview of the experimental setup. (B) RT-PCR of Ya1 transcripts in aphids and aphid-exposed plants. The 357-nt Ya1 transcript was found in aphids, and not in plants. A 273-nt transcript was found at aphid feeding sites and to migrate systemically in plants. A. thaliana actin 2 (AtActin2) and M. persicae elongation factor (MpEF) were used to control for presence of RNA. (C) Northern blot hybridizations with a Ya1 probe to detect Ya transcripts in aphids and plants. Arrows at right of the blots indicate the locations of the in vitro synthesized 291-nt Ya1-SP6 RNA transcript and Ya transcripts found in aphids and plants. Blots were stripped and labelled with the $A$. thaliana U6 probe to assess RNA loading levels among the samples. (D, E) Quantitative RT-PCR to detect systemic migration of $M$. persicae in (D) A. thaliana and (E) B. rapa, P. sativum, and Z. mays. Y-axes, Ya1 concentrations based on a standard curve (Suppl. Fig. S13). ND, not detected. Bars indicate mean \pm standard deviation (SD) of concentrations of Ya1 and two independent biological replicates. Different letters above the bars indicate significant differences between groups ( $p$ value $<0.01$, Student $t$ test). 
A

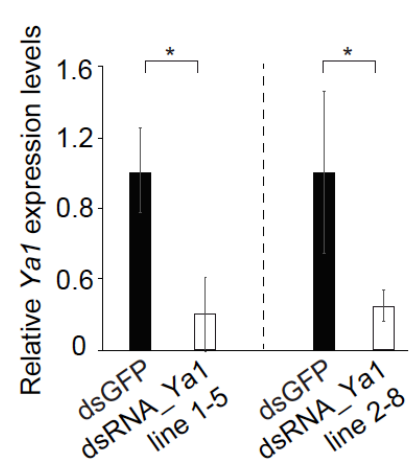

D

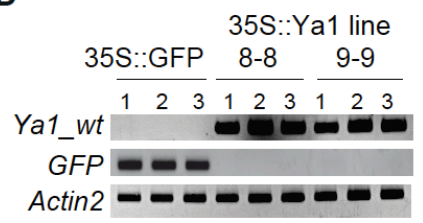

B

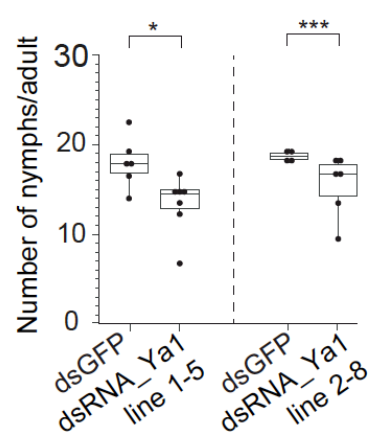

35S::Ya1_3ATG_mut line 35S::GFP $\frac{1-1}{12+3} \frac{3-3}{1+23} \frac{4-4}{1+3}$

C

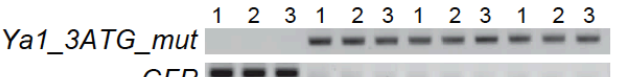

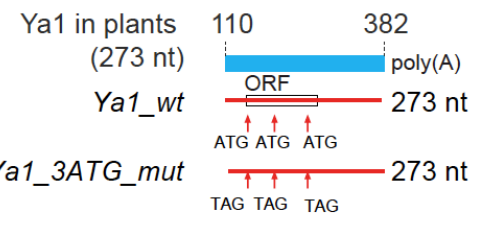

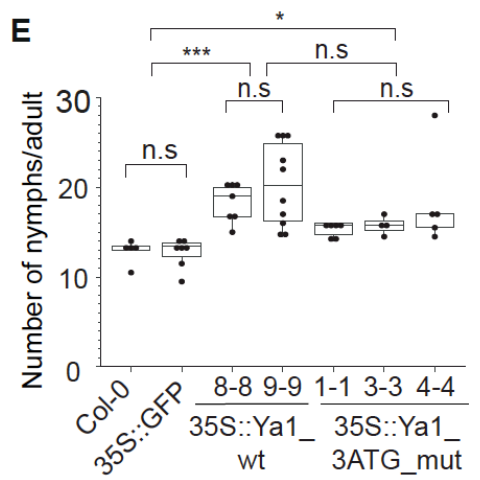

Fig. 5. M. persicae Ya IncRNA promotes $M$. persicae colonization on $A$. thaliana. (A) Quantitative RT-PCRs showing knock-down of Ya1 in M. persicae reared on plants that stably produce dsRNA_Ya1 relatively to those that produce dsRNA_GFP (controls). $\mathrm{Y}$-axis indicates the Ya1 expression levels relative to $M$. persicae EF reference gene. Bars represent relative mean \pm standard deviation (SD) expression values at $\mathrm{n}=4-8$ in which $\mathrm{n}$ is one aphid per plant. ${ }^{*} p<0.01$, Student $t$ test. (B) Reduction in fecundity of Ya1-RNAi aphids tested for Ya1 knock down in A. Each data point (black dot) represents the number of nymphs produced by one adult female per plant. Box plots show distribution of data points collected from $n=4-8$ female aphids per $A$. thaliana line as shown on the x-axis. (C) Mutation of the three ATGs within the ORF of 38-aa of the Ya1 transcript into TAG stop codons. (D) Detection of transgene transcripts by RT-PCRs at three plants per lines (1-3). Detection of the $A$. thaliana Actin2 transcript was used as control for presence of RNA. (E) Stable expression of Ya1_wt and Ya1_3ATG promotes M. persicae reproduction on plants. Box plots show distribution of nymphs produced by an adult female aphid per plant (black dots) collected from $n=4-10$ female aphids per $A$. thaliana line. ${ }^{* *} p<0.05$, ${ }^{* * *} p<0.001$, ANOVA followed by a Tukey-Kramer post-hoc test. 